OPEN ACCESS

Edited by:

Ademuyiwa S. Aromolaran, Masonic Medical Research Institute

(MMRI), United States

Reviewed by:

John Pearce Morrow,

Columbia University, United States

Przemyslaw Radwanski,

The Ohio State University,

United States

*Correspondence:

Lukáš Nalos

lukas.nalos@lfp.cuni.cz

Specialty section

This article was submitted to

Cardiac Electrophysiology,

a section of the journal

Frontiers in Physiology

Received: 09 April 2021

Accepted: 17 May 2021

Published: 21 June 2021

Citation:

Nalos L, Jarkovská $D$

Švíglerová J, Süß A, Záleský J,

Rajdl D, Krejčová M, Kuncová J,

Rosenberg J and Štengl M (2021)

TdP Incidence

in Methoxamine-Sensitized Rabbit Model Is Reduced With Age but Not

Influenced by Hypercholesterolemia.

Front. Physiol. 12:692921.

doi: 10.3389/fphys.2021.692921

\section{TdP Incidence in}

\section{Methoxamine-Sensitized Rabbit} Model Is Reduced With Age but Not Influenced by Hypercholesterolemia

\author{
Lukáš Nalos 1,2*, Dagmar Jarkovská1,2, Jitka Švíglerová1,2, Annabell Süß1, Jakub Záleský1, \\ Daniel Rajdl ${ }^{3}$, Milada Krejčová4, Jitka Kuncová ${ }^{1,2}$, Josef Rosenberg ${ }^{4}$ and Milan Štengl1,2

\begin{abstract}
${ }^{1}$ Department of Physiology, Faculty of Medicine in Pilsen, Charles University, Pilsen, Czechia, ${ }^{2}$ Biomedical Center, Faculty of Medicine in Pilsen, Charles University, Pilsen, Czechia, ${ }^{3}$ Institute of Clinical Biochemistry and Haematology, Faculty of Medicine in Pilsen, Charles University, Pilsen, Czechia, ${ }^{4}$ New Technologies for the Information Society, Faculty of Applied Sciences, University of West Bohemia, Pilsen, Czechia
\end{abstract}

Metabolic syndrome is associated with hypercholesterolemia, cardiac remodeling, and increased susceptibility to ventricular arrhythmias. Effects of diet-induced hypercholesterolemia on susceptibility to torsades de pointes arrhythmias (TdP) together with potential indicators of arrhythmic risk were investigated in three experimental groups of Carlsson's rabbit model: (1) young rabbits (YC, young control, age 12-16 weeks), older rabbits (AC, adult control, age 20-24 weeks), and older age-matched cholesterol-fed rabbits ( $\mathrm{CH}$, cholesterol, age 20-24 weeks). TdP was induced by $\alpha$-adrenergic stimulation by methoxamine and $\mathrm{I}_{\mathrm{Kr}}$ block in $83 \%$ of $\mathrm{YC}$ rabbits, $18 \%$ of $\mathrm{AC}$ rabbits, and $21 \%$ of $\mathrm{CH}$ rabbits. High incidence of $\mathrm{TdP}$ was associated with high incidence of single (SEB) and multiple ectopic beats (MEB), but the QTc prolongation and short-term variability (STV) were similar in all three groups. In TdP-susceptible rabbits, STV was significantly higher compared with arrhythmia-free rabbits but not with rabbits with other than TdP arrhythmias (SEB, MEB). Amplitude-aware permutation entropy analysis of baseline ECG could identify arrhythmia-resistant animals with high sensitivity and specificity. The data indicate that the TdP susceptibility in methoxamine-sensitized rabbits is affected by the age of rabbits but probably not by hypercholesterolemia. Entropy analysis could potentially stratify the arrhythmic risk and identify the low-risk individuals. Keywords: hypercholesterolemia, TdP arrhythmia, arrhythmia risk prediction, rabbit, amplitude-aware
permutation entropy

\section{INTRODUCTION}

Torsades de pointes arrhythmia (TdP) is a potentially life-threatening polymorphic ventricular tachycardia arising on the basis of QT interval prolongation. QT interval prolongation is a manifestation of decreased repolarization reserve, which comprises a substrate for TdP arrhythmia (Roden, 1998). Deteriorated repolarization reserve is associated with several congenital syndromes; acquired conditions, such as ischemia or heart failure; and also a wide variety of medications 
(Antoniou et al., 2017). Many drugs were withdrawn from the market or their development was terminated because of their potential to induce $\mathrm{TdP}$ or prolong QT interval (Redfern et al., 2003; Valentin, 2010). Metabolic syndrome is associated with abnormal electrical activities in the myocardium characterized by prolonged QT interval in the ECG, QT interval dispersion, and ventricular arrhythmias (Soydinc et al., 2006; Li et al., 2009; Karaagac et al., 2014; Park and Lee, 2018). Hypercholesterolemia induces electrical remodeling of cardiomyocytes and sympathetic nerve sprouting leading to increased vulnerability of the heart to ventricular fibrillations (Chen et al., 2001; Liu et al., 2009). Hyperlipidemia has a direct prolonging effect on action potential duration (Liu et al., 2009) and QT/QTc interval (Szabó et al., 2005). Treatment with statins lowers cardiovascular risk by reduction of QTc dispersion and ventricular premature complexes (Gualdiero et al., 2002; Vedre et al., 2009) and prevents prolongation of action potential duration (Liu et al., 2009). Despite an apparent link between hypercholesterolemia, QT prolongation, and TdP, little is known about the effect of hypercholesterolemia on $\mathrm{TdP}$ arrhythmia inducibility. Most published results concerning hypercholesterolemia and arrhythmogenicity came from models combining high cholesterol and high triglyceride plasma levels, and arrhythmia models were limited to rapid pacing-induced ventricular fibrillations or arrhythmias due to myocardial ischemia/reperfusion injury. To investigate the effects of hypercholesterolemia on long-QT arrhythmia inducibility, the Carlsson's methoxamine sensitized rabbit model of TdP arrhythmias (Carlsson et al., 1990) was combined with diet-induced hypercholesterolemia.

Age of the rabbits used for the Carlsson's model of TdP arrhythmias is usually between 12 and 16 weeks as these animals are considered to be adult (de Turckheim et al., 1983). Because rabbits in our study were fed with a cholesterol diet for 8-12 weeks, an additional age-matched group was included resulting in three experimental groups: (1) young rabbits (aged 12-16 weeks, comparable to other published data), (2) cholesterol-fed rabbits (aged 20-24 weeks), and (3) agematched rabbits (aged 20-24 weeks).

Despite enormous scientific effort, the prediction of (TdP) arrhythmia risk remains doubtful (Schwartz and Woosley, 2016). It became apparent that the simple prolongation of the QT interval is not a reliable indicator of arrhythmic risk (De Vecchis et al., 2018). Some spatial and temporal heterogeneity criteria, such as index of cardiac electrophysiological balance (Lu et al., 2013), prolongation of the interval between the peak and the end of the $\mathrm{T}$ wave (Tpeak to Tend) on the 12-lead ECG (Panikkath et al., 2011; Delinière et al., 2019), or beat-to-beat variability of repolarization (BVR) quantified as short-term variability (STV; Bossu et al., 2017) were recently introduced as potential risk indicators. Arrhythmia risk prediction by these methods, however, relies on analysis of the ECG signal preceding shortly the arrhythmic event and usually in the presence of pharmacological challenge. Therefore, in our study, we tried to enhance the predictive power by using entropy-based methods for analyzing baseline ECG prior to any challenge.
In general, analysis of entropy might reveal tiny irregularities of the ECG signal that perhaps occur in rabbits susceptible to TdP after pharmacological challenge. Abilities of permutation entropy (Bandt and Pompe, 2002) to capture the underlying dynamics of the time series are shown in a diversity of fields, including medicine (Olofsen et al., 2008). In our study, two recently introduced approaches for analysis of time series were used: (1) new empirical amplitude-aware permutation entropy (AAPE) was adopted to overcome the lack of amplitude consideration in standard permutation entropy analysis (Unakafova, 2015; Azami and Escudero, 2016; CuestaFrau et al., 2019); (2) information exergy index (IEIN) combines singular value decomposition and information exergy and allows capturing of the time development of irregularities (Zhang et al., 2013). These choices were based on comparative analysis of various techniques of time series analysis, such as complexity (Rosso et al., 2007), ergodicity, and mixing (Crutchfield and Feldman, 2003); detrended fluctuation analysis (Peng et al., 1994); and amplitude- and fluctuation-based dispersion entropy (Azami and Escudero, 2018).

\section{MATERIALS AND METHODS}

\section{Animals}

The study was conducted according to the guidelines of the Declaration of Helsinki and approved by the Committee for Experiments on Animals of the Charles University Faculty of Medicine in Pilsen and by the Ministry of Education, Youth, and Sports of the Czech Republic (protocol number MSMT47402/2012-30). Only female rabbits were used in order to avoid gender variability and because higher susceptibility to TdP arrhythmias was reported for both female patients and rabbits (e.g., Ebert et al., 1998). 37 female New Zealand white rabbits were divided into three groups: (1) young (juvenile) control rabbits fed standard chow (YC; $n=12$; age 12 16 weeks, and mean weight $2.4 \mathrm{~kg}$ ), (2) adult control rabbits fed standard chow (AC; $n=11$; age 20-24 weeks, and mean weight $4.1 \mathrm{~kg}$ ), and (3) adult hypercholesterolemic rabbits fed standard chow supplemented with $1 \%$ cholesterol for 8 12 weeks $(\mathrm{CH} ; n=14$; age $20-24$ weeks, and mean weight $4 \mathrm{~kg}$ ). All rabbits were fed ad libitum. Content of cholesterol in standard chow was below $0.01 \%$. Serum levels of total cholesterol, HDL cholesterol, and triglycerides were examined. Levels of total cholesterol, HDL-cholesterol, and triglycerides were measured in a clinical laboratory with routinely used enzymatic colorimetric methods by Roche on an automatic analyzer by Roche (Cobas c 8000, Basel, Switzerland). Shortly, total cholesterol was determined with the cholesterol oxidase method and HDL-cholesterol with cholesterol esterase and oxidase after masking non-HDL particles and triglycerides with a series of enzymatic reactions (lipoprotein lipase, glycerol kinase, and glycerolphosphate oxidase). Formation of a colored substance that is photometrically detected in all three methods is based on oxidation of a substrate with hydrogen peroxide; reaction is catalyzed by peroxidase. LDL cholesterol was calculated with the Friedewald equation $(\mathrm{LDL}=\mathrm{TC}-\mathrm{HDL}-\mathrm{TAG} / 2,2)$. 


\section{In vivo Experiments}

The methoxamine-sensitized rabbit model of TdP arrhythmia as described by Carlsson et al. (1990) was employed with minor modifications. Anesthesia was induced and maintained with ketamine $(35 \mathrm{mg} / \mathrm{kg})$ and xylazine $(5 \mathrm{mg} / \mathrm{kg})$ applied every $30 \mathrm{~min}$ i.m. The external jugular vein was exposed and cannulated for drug application. An ECG lead II was recorded using needle electrodes (Biopac System; Biopac Systems Inc, Goleta, CA, United States). After $10 \mathrm{~min}$ of recovery from surgery, control ECG was measured for $10 \mathrm{~min}$. Then application of methoxamine $(15 \mu \mathrm{g} / \mathrm{kg} / \mathrm{min}, 2 \mathrm{ml} / \mathrm{h})$ was started and continued for another $10 \mathrm{~min}$. This was followed by application of lower-dose dofetilide $(10 \mu \mathrm{g} / \mathrm{kg} / \mathrm{min}, 2 \mathrm{ml} / \mathrm{h})$ for $20 \mathrm{~min}$ on top of methoxamine. If no TdP arrhythmia developed, a higher dose of dofetilide $(20 \mu \mathrm{g} / \mathrm{kg} / \mathrm{min}, 4 \mathrm{ml} / \mathrm{h})$ was administered. The experiment was terminated after $20 \mathrm{~min}$ on a higher dose of dofetilide or after $20 \mathrm{~s}$ of persistent $\mathrm{TdP}$ arrhythmia. In dofetilide groups, data from recordings at the higher dose of dofetilide were analyzed and reported unless otherwise stated.

\section{Solutions and Chemicals}

Dofetilide was initially dissolved in $0.1 \mathrm{mM} \mathrm{HCl}(25 \mathrm{mg} / \mathrm{ml})$ and diluted in $0.9 \%$ saline to the required concentration. Methoxamine $\mathrm{HCl}$ was dissolved and diluted in $0.9 \%$ saline. All chemicals were purchased from Sigma Aldrich.

\section{Calculations}

ECG data were first analyzed manually in Biopac Student Lab 4.1 (Biopac System; Biopac Systems Inc, Goleta, CA, United States). The animals were considered TdP inducible when at least three TdP episodes were observed or when a TdP episode lasted more than $10 \mathrm{~s}$. TdP was defined as a polymorphic ventricular tachyarrhythmia with at least five consecutive undulating QRS complexes with a typical twisting around the isoelectric line of the ECG. We analyzed single ectopic beats (SEB), multiple ectopic beats (MEB), and bigeminy. MEB was defined as series of two to four ectopic beats. In one animal, atrio-ventricular block occurred. For automatic ECG analysis, in-house made software was used. Heart rate-corrected QT values were calculated using the formula especially developed for this animal model (Carlsson et al., 1993). BVR was determined as a STV: 30 consecutive beats were used for the calculation

$$
S T V=\sum \frac{\left|D_{n-1}-D_{n}\right|}{30 \sqrt{2}}
$$

where $D$ represents QTc (Thomsen et al., 2004).

The heart rate variability (HRV) was analyzed in MATLAB 2014b (MathWorks Inc., Natick, MA, United States, 2014) in intervals of the last $5 \mathrm{~min}$ of the baseline recording. $\mathrm{R}$ peaks were detected by the derivative-threshold algorithm (Pan and Tompkins, 1985). Ectopic beats were determined automatically (difference more than $20 \%$ from the mean RRinterval) and replaced using linear interpolation. The LombScargle periodogram (Lomb, 1976; Scargle, 1982; Press and Rybicki, 1989) was applied to estimate the power spectral density. Power in the low (LF; $0.04-0.15 \mathrm{~Hz}$ ) and high (HF; $0.15-0.40 \mathrm{~Hz}$ ) frequency range were calculated.
For entropy analysis of the ECG signal, a Matlab code was developed (MATLAB R2017a; MathWorks Inc., Natick, MA, United States, 2014) using the algorithms for calculation of AAPE (Azami and Escudero, 2016) and IEIN (Zhang et al., 2014) based on robust empirical permutation entropy rePEnew (Unakafova, 2015). Analysis of each ECG record provided values of AAPE and IEIN $\sigma$ (Tian et al., 2018). The first parameter characterizes the (ir)regularity of the ECG signal, whereas the second one describes its development in time. Sections of ECG records without artifacts were selected for analysis.

\section{Statistical Analysis}

Statistical analysis was performed using Origin 2017 (OriginLab Corp., Northampton, MA, United States). Data are presented as the mean $\pm \mathrm{SD}$. Statistical comparisons were made with one- or two-way repeated-measures ANOVA followed by post hoc Tukey test where appropriate. Outliers were identified using Grubbs test. Incidence was tested with the two-sample proportion test. Differences at $p \leq 0.05$ were considered to be significant.

\section{RESULTS}

In $\mathrm{CH}$, plasma levels of total cholesterol (baseline $1.85 \pm 0.42$ vs. $67 \pm 19.6 \mathrm{mmol} / \mathrm{L}$ after 8 weeks of diet) as well as of HDL cholesterol (baseline $0.85 \pm 0.2$ vs. $5.8 \pm 1.16 \mathrm{mmol} / \mathrm{L}$ after 8 weeks of diet) increased significantly, whereas the levels of triglycerides remained stable throughout the experiment (Figure 1A). LDL cholesterol was significantly increased in the $\mathrm{CH}$ group (baseline $0.57 \pm 0.32$ vs. $61 \pm 22.3 \mathrm{mmol} / \mathrm{L}$ after 8 weeks of diet). In AC the plasma levels of TC, HDL, LDL, and TAG were comparable to those at baseline in $\mathrm{CH}$ group and did not change significantly throughout the experiment (total cholesterol: baseline $1.68 \pm 0.47$ vs. $1.65 \pm 0.24 \mathrm{mmol} / \mathrm{L}$ after 8 weeks of normal diet, HDL cholesterol: $0.91 \pm 0.12$ vs. $1.1 \pm 0.16 \mathrm{mmol} / \mathrm{L}, \mathrm{LDL}$ cholesterol: $0.51 \pm 0.3$ vs. $0.39 \pm 0.17 \mathrm{mmol} / \mathrm{L}$, and triglycerides: $0.8 \pm 0.3 \mathrm{vs.}$ $0.36 \pm 0.03 \mathrm{mmol} / \mathrm{L})$

In the YC group, TdP arrhythmia could be induced in 10 out of 12 animals in the presence of a higher dose of dofetilide (inducibility $83 \%$, Figures $1 \mathrm{~B}, \mathrm{C}$ ). In the $\mathrm{AC}$ group, TdP only occurred in 2 out of 11 animals in the presence of a higher dose of dofetilide (inducibility 18\%, Figure 1B). In the $\mathrm{CH}$ group, TdP were observed in 3 out of 14 animals in the presence of a higher dose of dofetilide (inducibility $21 \%$, Figure 1B). Besides TdP arrhythmias, other arrhythmic events (SEB and/or MEB, bigeminy) occurred in all three groups: YC 12/12, 100\%; AC 7/11, $64 \%$; and $\mathrm{CH} 6 / 14,43 \%$ (Figure 1B).

Baseline ECG parameters were not significantly different between the groups (Table 1 and Figure 2C). During infusion of methoxamine, the RR interval was prolonged significantly in $\mathrm{YC}$ and $\mathrm{AC}$, but reflex bradycardia was not significant in the $\mathrm{CH}$ group (Table 1 and Figure 2A). Other ECG intervals remained unchanged (Table 1), and no arrhythmias were observed. Application of dofetilide further increased RR intervals (significant to baseline) and induced significant dose-dependent 
A

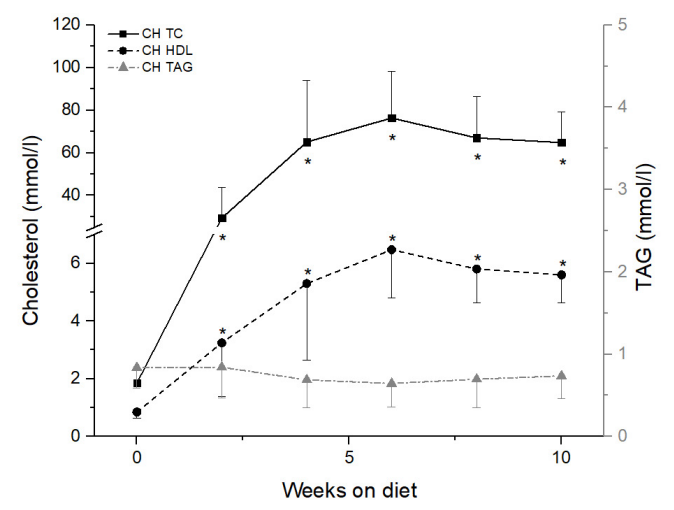

B

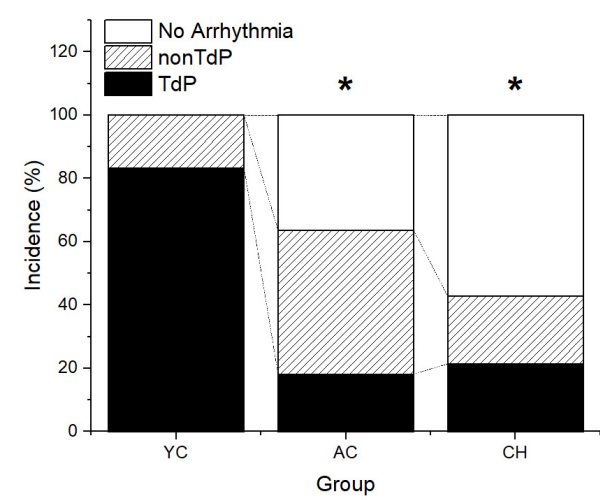

C

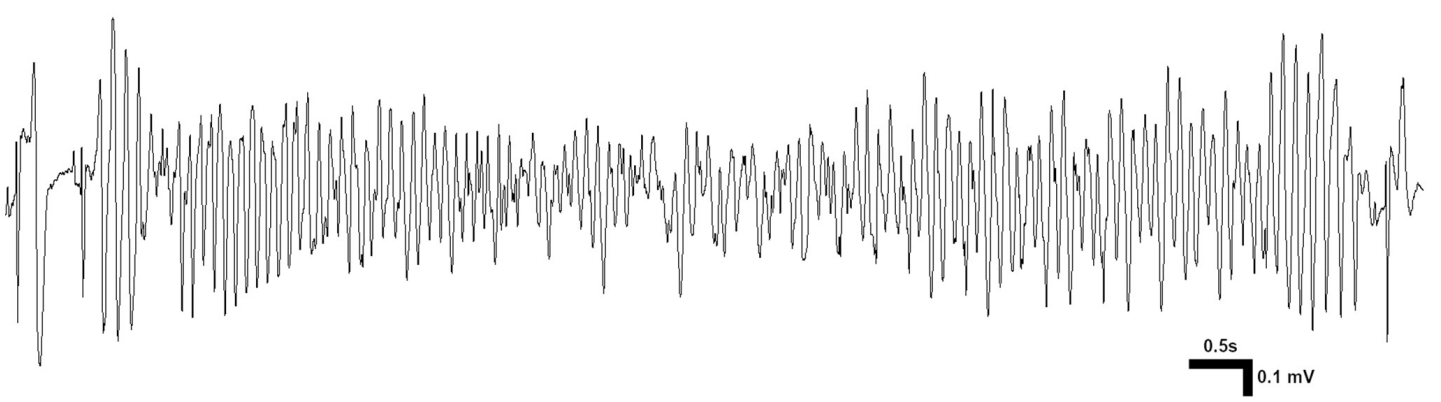

FIGURE 1 | (A) Total cholesterol (TC), HDL cholesterol (HDL), and triacylglycerol (TAG) plasma levels in cholesterol-fed rabbits (CH). ${ }^{*} P \leq 0.05$ vs. baseline. (B) Incidence of TdP and non-TdP arrhythmias in young rabbits (YC), adult control rabbits (AC), and cholesterol-fed rabbits (CH). ${ }^{*} p \leq 0.05$ vs. YC. (C) Representative recording of TdP arrhythmia.

TABLE 1 | ECG parameters of the rabbits

\begin{tabular}{|c|c|c|c|c|c|c|c|c|c|}
\hline & \multicolumn{3}{|c|}{ Control } & \multicolumn{3}{|c|}{ Methoxamine } & \multicolumn{3}{|c|}{ Methoxamine + Dofetilide } \\
\hline & YC & AC & $\mathrm{CH}$ & YC & AC & $\mathrm{CH}$ & YC & AC & $\mathrm{CH}$ \\
\hline $\mathrm{PQ}[\mathrm{msec}]$ & $68 \pm 4$ & $73 \pm 4$ & $72 \pm 5$ & $73 \pm 5^{\$}$ & $73 \pm 3$ & $74 \pm 5$ & $71 \pm 5$ & $83 \pm 7^{\star \#}$ & $86 \pm 22$ \\
\hline QRS [msec] & $68 \pm 5$ & $75 \pm 6$ & $74 \pm 6$ & $71 \pm 5^{\$}$ & $76 \pm 6$ & $77 \pm 8^{\$}$ & $78 \pm 5$ & $95 \pm 19^{\text {*\# }}$ & $87 \pm 14$ \\
\hline QT [msec] & $220 \pm 26$ & $225 \pm 26$ & $205 \pm 21$ & $250 \pm 24$ & $250 \pm 31$ & $228 \pm 43$ & $312 \pm 19^{\star \#}$ & $324 \pm 46^{\star \#}$ & $297 \pm 30^{\star \#}$ \\
\hline
\end{tabular}

$Y C$, young control group; $\mathrm{AC}$, adult control group; and $\mathrm{CH}$, cholesterol fed group. ${ }^{*} p \leq 0.05$ vs. Control, ${ }^{\#} p \leq 0.05$ vs. Methoxamine, and ${ }^{\$} p \leq 0.05$ vs. AC group.

prolongation of the QTc interval in all groups (Table 1 and Figure 2B).

Because the differences in inducibility between young and adult rabbits could possibly be attributed to different autonomic nervous system drive, the frequency domain analysis of the HRV was performed to quantify changes in autonomic regulation. No significant difference between the groups were found for both high- and low-frequency bands (Figure 3A). STV of QTc interval (STV) was significantly increased in the presence of dofetilide only in the CH group (Figure 3B). Occurrences of SEB or MEB were manually evaluated in ECG recordings in the presence of dofetilide prior to TdP arrhythmia (Figure 3C). Incidence of SEB and MEB was significantly higher in the YC group compared with the $\mathrm{AC}$ and $\mathrm{CH}$ groups.
In search of predictive indicators of TdP arrhythmia risk, the animals were regrouped into groups of TdP responders $(n=14)$, in which TdP arrhythmias developed, and nonresponders without TdP arrhythmia $(n=23)$, which were further subdivided into the group of no arrhythmias $(n=12)$ without any arrhythmic events and a group of non-TdP arrhythmias $(n=11)$, in which arrhythmic events other than TdP (SEB, MEB, and bigeminy) were observed. In all three groups, QTc was significantly prolonged only in the presence of dofetilide, and there was no significant difference between the groups (Figure 4A). Analysis of HRV at baseline revealed a significantly lower HF component and consequently higher LF/HF ratio in the group of TdP responders when compared with the group of no arrhythmias (Figure 4B). STV increased significantly 


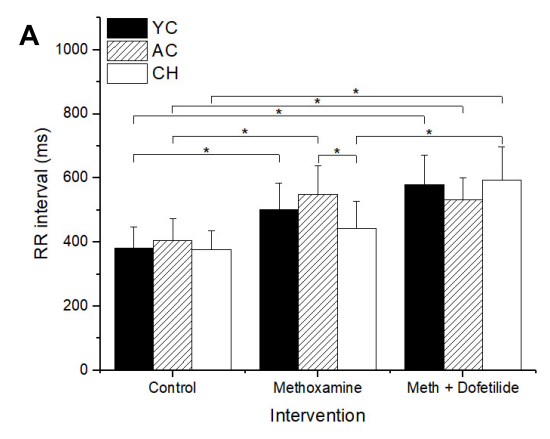

C

Control<smiles>CCCCCCCCCCCCCCCCCCCCCC</smiles><smiles>CCCCCC(CCCC)C(CCC)C(=O)O</smiles>

YC

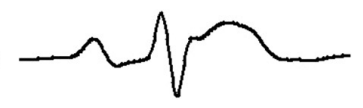

Dofetilide
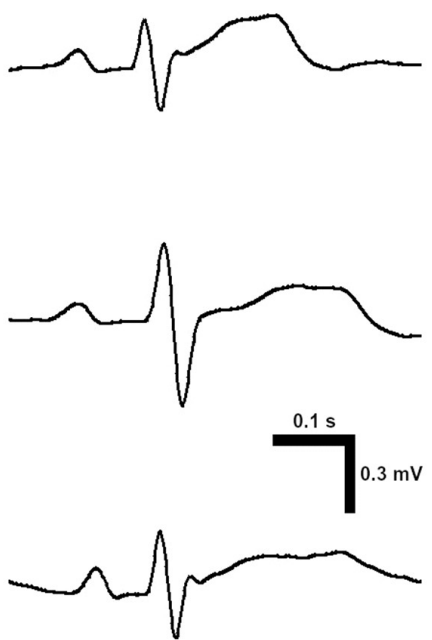

FIGURE 2 | ECG characteristics of young rabbits $(Y C)$, adult control rabbits $(A C)$, and cholesterol-fed rabbits $(C H)$ at baseline, in the presence of methoxamine, and in the presence of methoxamine + dofetilide. ${ }^{*} p \leq 0.05$. (A) RR interval, (B) QTc interval. (C) Representative examples of ECG.

A

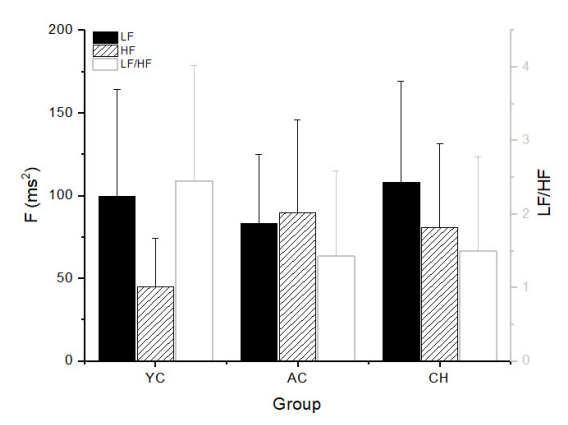

B

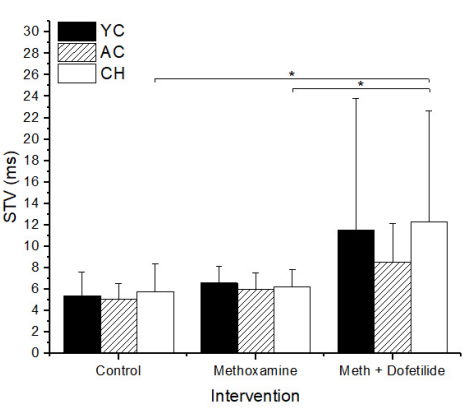

C

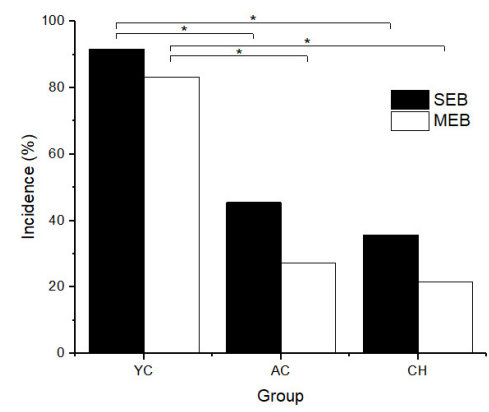

FIGURE 3 | Autonomic drive, STV and ectopic activity in young (YC), adult control (AC), and cholesterol-fed rabbits (CH). (A) HRV expressed as power in the low (LF) and high (HF) frequency bands and ratio of LF/HF (LF/HF). (B) Short term variability (STV) of QTc. (C) Incidence of single (SEB) and multiple (MEB) ectopic beats. ${ }^{*} p \leq 0.05$.

only in the group of $\mathrm{TdP}$ responders in the presence of dofetilide when it was significantly higher than in the group of no arrhythmias but not the group of non-TdP arrhythmias (Figure 4C). Incidence of $\mathrm{SEB}$ and $\mathrm{MEB}$ in the presence of dofetilide were significantly higher in the group of TdP responders (Figure 4D) providing $\mathrm{TdP}$ prediction sensitivity of 1 and 0.86 and specificity of 0.7 and 0.83 for SEB and $\mathrm{MEB}$, respectively.

Not all recordings had enough long sections without artifacts for entropy analysis, so only ECG recordings of 31 rabbits could be analyzed. The sizes of groups were TdP responders, 10 rabbits; no arrhythmias group, 10 rabbits; and group of non-TdP arrhythmias, 11 rabbits. Analysis of entropy in baseline ECG recordings (without any pharmacological challenge on board) using AAPE and IEIN approaches revealed significantly lower entropy parameters in the group of no arrhythmias (Figures 5A,B). When both AAPE and IEIN were combined, the arrhythmia-free animals clustered in the bottom-left quadrant (Figure 5C). AAPE analysis allowed determination of low-risk (arrhythmia-free) animals with excellent sensitivity and specificity (sensitivity of 1 , specificity of 0.91 ; arbitrary threshold 1.975), whereas IEIN showed lower specificity (sensitivity of 1 , specificity of 0.53 ; arbitrary threshold 670). 
A

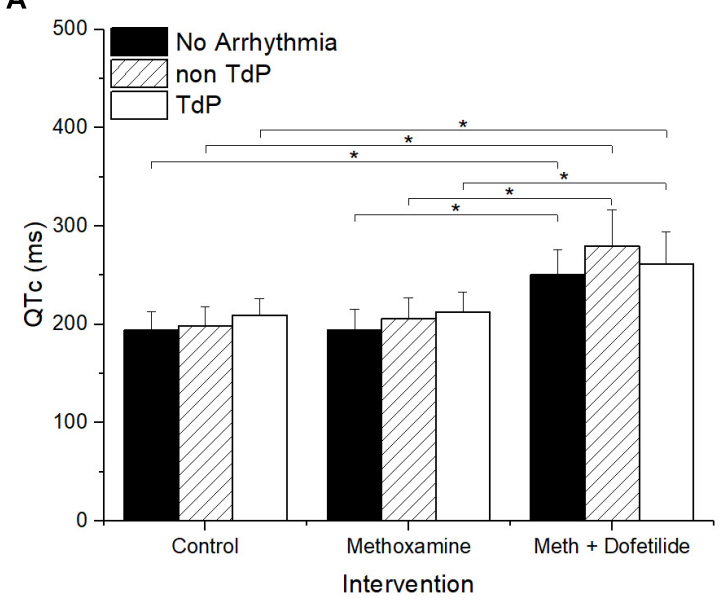

C

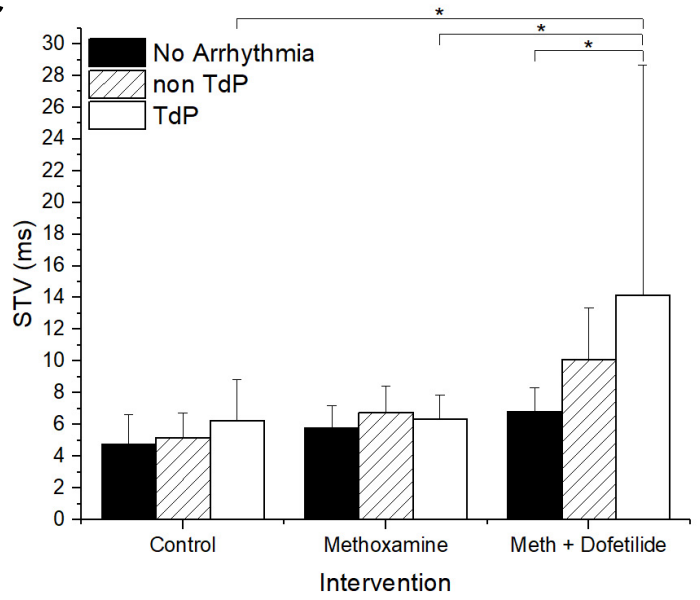

B

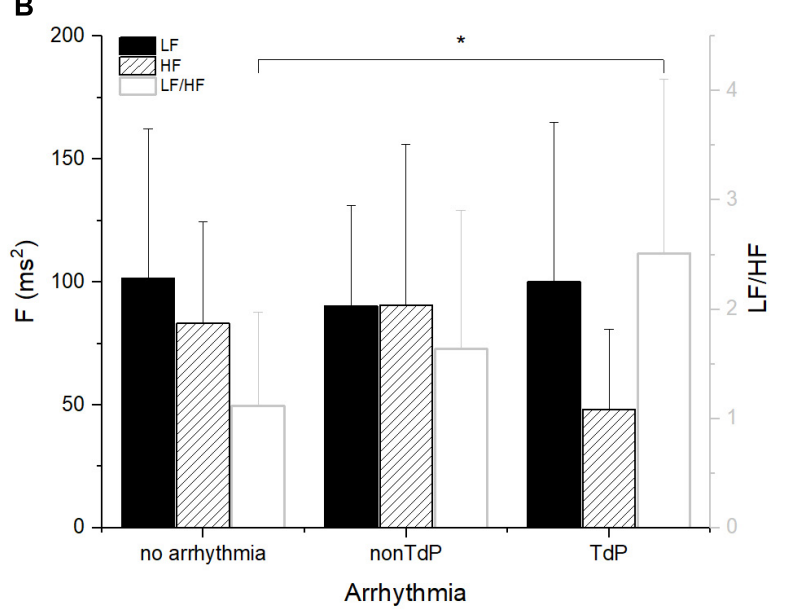

D

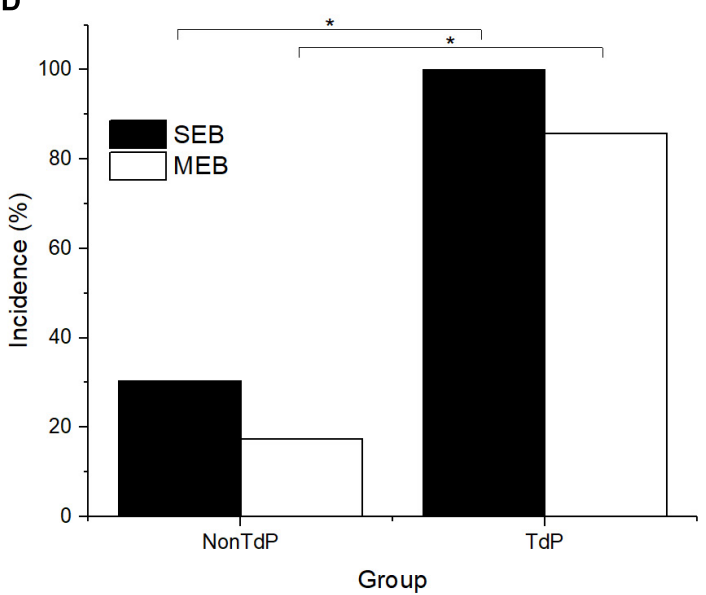

FIGURE 4 | QTC, autonomic drive, STV and ectopic activity in rabbits without arrhythmia (no arrhythmia), with non-TdP arrhythmias (non TdP), and with TdP arrhythmia (TdP). (A) QTc interval, (B) HRV expressed as power in the low (LF) and high (HF) frequency bands and ratio of LF/HF (LF/HF). (C) Short term variability (STV) of QTc. (D) Incidence of single (SEB) and multiple (MEB) ectopic beats. * $p \leq 0.05$.

A

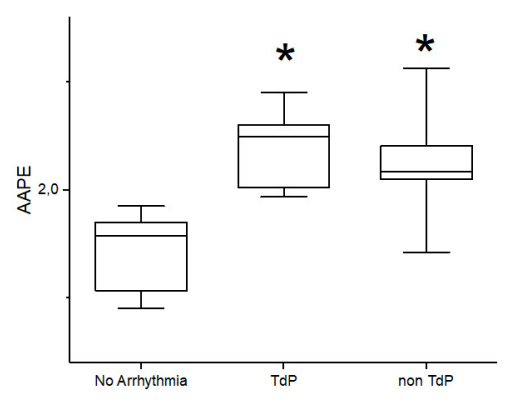

B

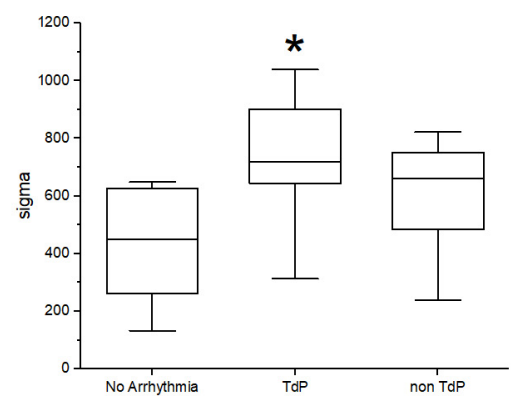

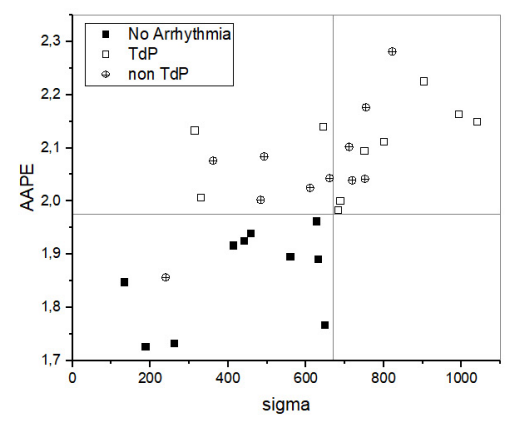

FIGURE 5 | Entropy analysis in rabbits without arrhythmia (no arrhythmia), with non TdP arrhythmia (non TdP), and with TdP arrhythmia (TdP). (A) Amplitude-aware permutation entropy (AAPE), (B) information exergy index (IEIN, sigma). (C) AAPE and IEIN relation; arbitrary threshold 1.975 and 670 , respectively. ${ }^{*} p \leq 0.05$ vs. no arrhythmia group. 


\section{DISCUSSION}

In the first part of the study, the influences of age and hypercholesterolemia on the susceptibility to TdP arrhythmias in a methoxamine-sensitized rabbit model were investigated. In the second part of the study, several indicators of proarrhythmic risk were analyzed to determine their predictive power.

\section{Effect of Age on TdP Inducibility}

In young rabbits (age 3-4 months), high susceptibility to TdP arrhythmias (TdP induced in $83 \%$ of young rabbits) comparable to earlier reports (e.g., Carlsson et al., 1990, 1993; Vincze et al., 2008) was observed, whereas in adult rabbits (age 5-6 months) the TdP inducibility was significantly lower regardless of plasma cholesterol levels (TdP induced in $18 \%$ of adult normocholesterolemic rabbits vs. $21 \%$ of adult hypercholesterolemic rabbits). Although the rabbits of age 3-4 months (weight of 2-3 kg) are often used in experimental research as adult animals, they should be rather considered peripubertal because puberty in rabbits occurs from 3 to 5 months of age (Kamwanja and Hauser, 1983; Laffan et al., 2018). Detailed ontogenetic studies focusing on cardiac development in rabbits within this age period (3-4 vs. 5-6 months) are missing; however, with regard to the peripubertal time window, effects of varying levels of sex hormones are likely. Progesterone and estrogens are reported to exert opposing effects on the heart. Progesterone pretreatment is shown to reduce the incidence of drug-induced TdP in atrioventricular node-ablated isolated perfused rabbit hearts (Tisdale et al., 2019) and to protect against prolongation of action potential duration - APD (90) and triangulation associated with potassium channel inhibition (Tisdale et al., 2011). In isolated guinea pig ventricular myocytes, progesterone shortened action potential duration, probably due to enhancement of the slow delayed rectifier $\mathrm{K}^{+}$current $\left(\mathrm{I}_{\mathrm{Ks}}\right)$ under basal conditions and inhibition of L-type $\mathrm{Ca}^{2+}$ currents $\left(\mathrm{I}_{\mathrm{CaL}}\right)$ under cAMPstimulated conditions (Nakamura et al., 2007). On the other hand, estradiol is demonstrated to potentiate QTc-prolonging effects of d,l-sotalol and to increase the susceptibility to d,1sotalol-induced arrhythmias (Cheng et al., 2012), dominant probably due to upregulation of L-type $\mathrm{Ca}^{2+}$ channels (Yang et al., 2012, 2018) with greater dispersion in $\mathrm{I}_{\mathrm{CaL}}$ density (Pham et al., 2002), reduced repolarization reserve, and enhanced $\mathrm{Ca}^{2+}$ overload (Sims et al., 2008). In transgenic long QT type 2 rabbits, estradiol promotes polymorphic ventricular tachyarrhythmias and sudden cardiac death although progesterone prevented them (Odening et al., 2012). Estradiol exerted the proarrhythmic effect by changing the arrhythmogenic substrate due to increased $\mathrm{I}_{\mathrm{Ks}}$ and $\mathrm{I}_{\mathrm{CaL}}$ currents, whereas progesterone, in contrast, exerted an antiarrhythmic effect by preventing early afterdepolarizations, likely due to an increase in SERCA2a and a decrease in the oscillatory $\mathrm{I}_{\mathrm{CaL}}$ current (Odening et al., 2012). With regard to the opposing effects of estrogens and progesterone, slight changes in the progesterone:estrogen ratio may have profound consequences for cardiac repolarization and arrhythmia vulnerability as indicated by variable drug-induced QT prolongation in women during the menstrual cycle (Rodriguez et al., 2001).
In contrast to our findings, an earlier study of similar experimental design revealed no significant gender- and agedependent differences in TdP incidence in a rabbit model of the acquired long QT syndrome (Johansson and Carlsson, 2001). Several factors probably contributed to this discrepancy: slightly lower age of rabbits in both young and adult groups (on average by 2 weeks), different anesthesia (methohexital and $\alpha$-chloralose), and different $\mathrm{I}_{\mathrm{Kr}}$ blocker (ibutilide). In contrast to dofetilide (used in our study), ibutilide in guinea pig cardiac myocytes at low concentrations increased the late inward current, and at high concentrations it increased the outward current (Lee et al., 1993). Consequently, ibutilide could, depending on the concentration, either enhance or depress the effect of $\mathrm{K}^{+}$channel blockers on action potential duration.

\section{Effect of Hypercholesterolemia on TdP Inducibility}

In our study, high cholesterol plasma levels did not influence susceptibility to TdP arrhythmias in the age-matched groups of the methoxamine-sensitized rabbit model. Levels of both non-HDL and HDL cholesterol were increased, whereas levels of triglycerides were comparable in both groups. Similarly, no association between HDL levels and ventricular repolarization indexes was found in subjects with primary hypercholesterolemia (Korantzopoulos et al., 2014). On the other hand, reconstituted HDL cholesterol shortened cardiac repolarization in isolated cardiomyocytes as well as in dyslipidemic patients (Den Ruijter et al., 2011) suggesting that decreased levels of HDL cholesterol (and not the elevated levels of total cholesterol) in patients with metabolic syndrome might contribute to QTC prolongation in these patients. In contrast to our results, pronounced neural and electrophysiological remodeling with nerve sprouting, prolonged action potential duration, longer QTc intervals, increased repolarization dispersion, and increased vulnerability to fibrillation were previously demonstrated in hypercholesterolemic rabbits (Liu et al., 2003). Both serum cholesterol levels and serum triglyceride levels were increased in these rabbits. Because, in our experiments, the cholesterol levels were increased to similar levels but triglycerides were not elevated, it is tempting to speculate that elevated plasma levels of triglycerides (but not of cholesterol) are necessary for inducing the proarrhythmic cardiac electrical remodeling.

\section{Autonomic Nervous System}

The autonomic nervous system is an important modulator of cardiac electrophysiology and arrhythmogenesis (Shen and Zipes, 2014) and the $\alpha_{1}$-adrenergic stimulation is a prerequisite of arrhythmia induction in the rabbit model. Analysis of HRV at baseline revealed higher LF/HF ratios in rabbits susceptible to TdP arrhythmias, dominantly due to the lower HF component. These findings suggest a shift of the sympathovagal balance to sympathetic dominance due to the diminished vagal drive in TdP-susceptible rabbits, which is in line with putative protective effects of vagal stimulation against malignant ventricular arrhythmias (Brack et al., 2011). The protective effect of vagus nerve stimulation in isolated rabbit hearts was attributed 
to direct nitrergic action in the ventricle of post-ganglionic intracardiac nerves activated by vagus nerve (Brack et al., 2011).

\section{TdP Arrhythmia Prediction}

To determine and predict the proarrhythmic risk, several electrophysiological parameters were analyzed and compared between groups of animals without any arrhythmia with $\mathrm{TdP}$ arrhythmias and with arrhythmias other than TdP (SEB, MEB, and bigeminy). In line with a number of previous studies (Thomsen et al., 2004), QTc intervals were not different between the groups, either at baseline or after challenge with $\mathrm{I}_{\mathrm{Kr}}$ block. In contrast to the low predictive power of QTc interval prolongation, temporal STV of ventricular repolarization was repeatedly reported as a promising indicator of proarrhythmic risk in both experimental (Thomsen et al., 2004; Smoczynska et al., 2019) and clinical settings (Hinterseer et al., 2010; Orosz et al., 2015). In our rabbit model, STV in TdP-susceptible rabbits was indeed significantly increased in the presence of $\mathrm{I}_{\mathrm{Kr}}$ block, and it was significantly different from the STV of rabbits without any arrhythmia. However, the interindividual variability in the group of TdP-susceptible rabbits was considerable, and the difference between the groups of TdP-susceptible rabbits and rabbits with other than TdP arrhythmias was not significant. It is questionable whether the insignificant difference in STV of TdP susceptible rabbits and rabbits with other than TdP arrhythmias was due to an insufficient predictive power of STV, or whether, in rabbits with other than $\mathrm{TdP}$ arrhythmias, a delayed development of TdP arrhythmias might occur (and these rabbits would, in reality, belong to the group of TdP-susceptible rabbits). The incidence of SEB and MEB was significantly higher in the group of TdP-susceptible rabbits, indicating their role in the genesis of TdP arrhythmias as suggested earlier (Verduyn et al., 1997). Both SEB and MEB showed rather high sensitivity but lower specificity. Anyway, it should be emphasized that both elevated STV and incidences of SEB and MEB only occurred in the presence of pharmacological challenge, IKr block, which considerably limits their potential clinical use.

Because analysis of entropy allows capturing the underlying dynamics of time series, it might possibly detect tiny irregularities of the ECG signal that reflect the differential susceptibility to arrhythmias even in baseline recordings. After testing various methods for analysis of time series, only methods based on entropy and sensitive to irregularities in amplitude revealed significant differences between baseline ECG records of arrhythmia susceptible and non-susceptible groups. Permutation entropy is a relatively simple time series complexity measure that is based on the Shannon information entropy and provides comparable results to other entropies, e.g., Kolmogorov-Sinai entropy (Unakafova, 2015). The standard permutation entropy analysis in our study, however, did not identify any significant differences between the groups, indicating that analyzing only the sample order but not the amplitude limits the power of the method as shown earlier (Cuesta-Frau, 2019). To overcome this amplitude-related limitation we have used the AAPE method as the most robust, simple, and effective method and, interestingly, it was able to distinguish the group without arrhythmias from other two arrhythmic groups.
Furthermore, the entropy-based methods rely on moment sensory condition monitoring time series, and the time-varying measurement uncertainty cannot be properly managed (Zhang et al., 2013). To reduce the uncertainty within the diagnostic process, especially the time-varying measurement uncertainty, an information exergy-based method integrating multipoint and multimoment monitoring information was adopted from the field of structural damage diagnosis (Zhang et al., 2013), and the IEIN algorithm was developed (Zhang et al., 2014). The combination of AAPE and IEIN methods led to clustering of arrhythmia-free animals within arbitrary boundaries of the bottom-left quadrant. The other two groups of rabbits with TdP arrhythmias and rabbits with other than TdP arrhythmias, however, showed similar distribution patterns and could not be distinguished. In line with STV measurements, this perhaps suggests that TdP would eventually develop in the group of rabbits with other than TdP arrhythmias. Anyway, the data indicate that the entropy analysis of baseline ECG recordings (without any pharmacological challenge) could potentially stratify the arrhythmic risk and at least identify the low-risk individuals. To the best of our knowledge, the AAPE and EIEN approaches are so far never used for analysis of clinical ECG (either 12-lead or Holter), and perhaps, the concept could be extended to more arrhythmic phenotypes. From technical point of view, the application of entropy-based approaches to a clinical level should be easily possible; defining optimal patient groups, however, requires collaboration with experienced clinical cardiologists.

\section{Study Limitations}

Statistically reliable analysis within individual age and cholesterol groups would require, with regard to the TdP incidence, rather high numbers of experimental animals $(\sim 50$ in each group). To reduce the number of animals used, we instead regrouped the animals according to arrhythmic endpoints, which allowed generalizing the predictive potential of the indicators across the experimental groups as required in clinical practice. Analysis of cardiac electrophysiological remodeling and proarrhythmic mechanisms on a cellular level would certainly add to understanding of the susceptibility or resistance to TdP arrhythmias in the rabbit model. It could also contribute to better distinction of the arrhythmic groups and elucidating whether the two arrhythmic groups show different TdP susceptibility due to intrinsic cellular (remodeling) differences or rather due to improper experimental design (e.g., a short duration of pharmacological challenge). Anyway, detailed analysis of cellular mechanisms of arrhythmia induction was beyond the scope of this study and will be addressed in future studies. In our study, only females were used, and gender differences cannot be excluded. LDL cholesterol levels were calculated with the Friedewald equation, which is routinely used in human medicine but was not verified for rabbits. Association between hypercholesterolemia and inflammation was not addressed in this study, and inflammatory markers were not measured. However, elevated CRP levels were described in a cholesterol-fed rabbit model previously (Yu et al., 2012), suggesting a good correlation with human pathology. 


\section{DATA AVAILABILITY STATEMENT}

The raw data supporting the conclusions of this article will be made available by the authors, without undue reservation.

\section{ETHICS STATEMENT}

The animal study was reviewed and approved by Committee for Experiments on Animals of the Charles University Faculty of Medicine in Pilsen and by the Ministry of Education, Youth, and Sports of the Czechia.

\section{AUTHOR CONTRIBUTIONS}

MŠ contributed to conception. MŠ and LN contributed to design of the study. LN, DJ, JŠ, AS, and JZ performed the experiments. DR performed cholesterol laboratory evaluation. JR and MK

\section{REFERENCES}

Antoniou, C. K., Dilaveris, P., Manolakou, P., Galanakos, S., Magkas, N., Gatzoulis, K., et al. (2017). QT prolongation and malignant arrhythmia: how serious a problem? Eur. Cardiol. Rev. 12, 112-120. doi: 10.15420/ecr.2017:16:1

Azami, H., and Escudero, J. (2016). Amplitude-aware permutation entropy: illustration in spike detection and signal segmentation. Comput. Methods Programs Biomed. 128, 40-51. doi: 10.1016/j.cmpb.2016.02.008

Azami, H., and Escudero, J. (2018). Amplitude- and fluctuation-based dispersion entropy. Entropy 20:210. doi: 10.3390/e20030210

Bandt, C., and Pompe, B. (2002). Permutation entropy: a natural complexity measure for time series. Phys. Rev. Lett. 88:174102. doi: 10.1103/PhysRevLett. 88.174102

Bossu, A., Varkevisser, R., Beekman, H. D. M., Houtman, M. J. C., van der Heyden, M. A. G., and Vos, M. A. (2017). Short-term variability of repolarization is superior to other repolarization parameters in the evaluation of diverse antiarrhythmic interventions in the chronic atrioventricular block dog. J. Cardiovasc. Pharmacol. 69, 398-407. doi: 10.1097/FJC.00000000000 00488

Brack, K. E., Coote, J. H., and Ng, G. A. (2011). Vagus nerve stimulation protects against ventricular fibrillation independent of muscarinic receptor activation. Cardiovasc. Res. 91, 437-446. doi: 10.1093/cvr/cvr105

Carlsson, L., Abrahamsson, C., Andersson, B., Duker, G., and Schiller-Linhardt, G. (1993). Proarrhythmic effects of the class III agent almokalant: importance of infusion rate, QT dispersion, and early afterdepolarisations. Cardiovasc. Res. 27, 2186-2193. doi: 10.1093/cvr/27.12.2186

Carlsson, L., Almgren, O., and Duker, G. (1990). QTU-prolongation and torsades de pointes induced by putative class III antiarrhythmic agents in the rabbit: etiology and interventions. J. Cardiovasc. Pharmacol. 16, 276-285. doi: 10.1097/ 00005344-199008000-00014

Chen, P. S., Chen, L. S., Cao, J. M., Sharifi, B., Karagueuzian, H. S., and Fishbein, M. C. (2001). Sympathetic nerve sprouting, electrical remodeling and the mechanisms of sudden cardiac death. Cardiovasc. Res. 50, 409-416. doi: 10. 1016/s0008-6363(00)00308-4

Cheng, J., su, D., ma, X., and li, H. (2012). Concurrent supplement of estradiol and progesterone reduces the cardiac sensitivity to D,L-sotalol-induced arrhythmias in ovariectomized rabbits. J. Cardiovasc. Pharmacol. Ther 17, 208-214. doi: 10.1177/1074248411418972

Crutchfield, J. P., and Feldman, D. P. (2003). Regularities unseen, randomness observed: levels of entropy convergence. Chaos 13, 25-54. doi: 10.1063/1. 1530990

Cuesta-Frau, D. (2019). Permutation entropy: influence of amplitude information on time series classification performance. Math. Biosci. Eng. 16, 6842-6857. doi: $10.3934 / \mathrm{mbe} .2019342$ performed entropy analysis. DJ performed automatic and LN manual analysis of ECG. LN and JK performed the statistical analysis. LN and MŠ wrote the first draft of the manuscript. JR and MK wrote sections of the manuscript. All authors contributed to manuscript revision, read, and approved the submitted version.

\section{FUNDING}

This research was funded by operational program ECOP "increasing of the R\&D capacity at Charles University through new positions for graduates of doctoral studies," grant number CZ.1.07/2.3.00/30.0061, program for the Development of Scientific Fields of Charles University, grant number Progres Q39 and European Regional Development Fund, project. "Application of Modern Technologies in Medicine and Industry" grant number CZ.02.1.01/0.0/0.0/17_048/0007280.

Cuesta-Frau, D., Murillo-Escobar, J. P., Orrego, D. A., and Delgado-Trejos, E. (2019). Embedded dimension and time series length. Practical influence on permutation entropy and its applications. Entropy 21:385. doi: 10.3390/ e21040385

de Turckheim, M., Berger, M., Jean-Faucher, C., Veyssière, G., and Jean, C. (1983). Changes in ovarian oestrogens and in plasma gonadotrophins in female rabbits from birth to adulthood. Acta Endocrinol. (Copenh) 103, 125-130. doi: 10.1530/ acta.0.1030125

De Vecchis, R., Ariano, C., Di Biase, G., and Noutsias, M. (2018). Acquired druginduced long QTc: new insights coming from a retrospective study. Eur. J. Clin. Pharmacol. 74, 1645-1651. doi: 10.1007/s00228-018-2537-y

Delinière, A., Baranchuk, A., Giai, J., Bessiere, F., Maucort-Boulch, D., Defaye, P., et al. (2019). Prediction of ventricular arrhythmias in patients with a spontaneous Brugada type 1 pattern: the key is in the electrocardiogram. Europace 21, 1400-1409. doi: 10.1093/europace/euz156

Den Ruijter, H. M., Franssen, R., Verkerk, A. O., van Wijk, D. F., Vaessen, S. F., Holleboom, A. G., et al. (2011). Reconstituted high-density lipoprotein shortens cardiac repolarization. J. Am. Coll. Cardiol. 58, 40-44. doi: 10.1016/j.jacc.2010. 11.072

Ebert, S. N., Liu, X. K. E., and Woosley, R. L. (1998). Female gender as a risk factor for drug-induced cardiac arrhythmias: evaluation of clinical and experimental evidence. J. Womens Health 7, 547-557. doi: 10.1089/jwh.1998.7.547

Gualdiero, P., Esposito, K., Ciotola, M., Marfella, R., and Giugliano, D. (2002). Simvastatin normalizes qtc dispersion and reduces ventricular electrical instability in isolated hypercholesterolemia. J. Endocrinol. Invest. 25, RC16RC18. doi: 10.1007/BF03345487

Hinterseer, M., Beckmann, B. M., Thomsen, M. B., Pfeufer, A., Ulbrich, M., Sinner, M. F., et al. (2010). Usefulness of short-term variability of QT intervals as a predictor for electrical remodeling and proarrhythmia in patients with nonischemic heart failure. Am. J. Cardiol. 106, 216-220. doi: 10.1016/j.amjcard. 2010.02.033

Johansson, M., and Carlsson, L. (2001). Female gender does not influence the magnitude of ibutilide-induced repolarization delay and incidence of torsades de pointes in an in vivo rabbit model of the acquired long QT syndrome. J. Cardiovasc. Pharmacol. Ther. 6, 247-254. doi: 10.1177/107424840100600305

Kamwanja, L. A., and Hauser, E. R. (1983). The influence of photoperiod on the onset of puberty in the female rabbit. J. Anim. Sci. 56, 1370-1375. doi: $10.2527 /$ jas $1983.5661370 \mathrm{x}$

Karaagac, K., Emul, A., Tenekecioglu, E., Agca, F. V., Ozluk, O. A., Tutuncu, A., et al. (2014). The effects of metabolic syndrome on TpTe interval and TpTe/QT ratio in patients with normal coronary arteries. Eurasian J. Med. 46, 182-186. doi: 10.5152/eajm.2014.48

Korantzopoulos, P., Liberopoulos, E., Barkas, F., Kei, A., Goudevenos, J. A., and Elisaf, M. (2014). No association between high-density lipoprotein 
levels and ventricular repolarization indexes in subjects with primary hypercholesterolemia. Scand. J. Clin. Lab. Invest. 74, 53-58. doi: 10.3109/ 00365513.2013.857041

Laffan, S. B., Posobiec, L. M., Uhl, J. E., and Vidal, J. D. (2018). Species comparison of postnatal development of the female reproductive system. Birth Defects Res. 110, 163-189. doi: 10.1002/bdr2.1132

Lee, K. S., Tsai, T. D., and Lee, E. W. (1993). Membrane activity of class III antiarrhythmic compounds; a comparison between ibutilide, d-sotalol, E-4031, sematilide and dofetilide. Eur. J. Pharmacol. 234, 43-53. doi: 10.1016/00142999(93)90704-L

Li, W., Bai, Y., Sun, K., Xue, H., Wang, Y., Song, X., et al. (2009). Patients with metabolic syndrome have prolonged corrected QT interval (QTc). Clin. Cardiol. 32, E93-E99. doi: 10.1002/clc.20416

Liu, Y. B., Lee, Y. T., Pak, H. N., Lin, S. F., Fishbein, M. C., Chen, L. S., et al. (2009). Effects of simvastatin on cardiac neural and electrophysiologic remodeling in rabbits with hypercholesterolemia. Heart Rhythm 6, 69-75. doi: 10.1016/j. hrthm.2008.10.004

Liu, Y. B., Wu, C. C., Lu, L. S., Su, M. J., Lin, C. W., Lin, S. F., et al. (2003). Sympathetic nerve sprouting, electrical remodeling, and increased vulnerability to ventricular fibrillation in hypercholesterolemic rabbits. Circ. Res. 92, 11451152. doi: 10.1161/01.RES.0000072999.51484.92

Lomb, N. R. (1976). Least-squares frequency analysis of unequally spaced data. Astrophys. Space Sci. 39, 447-462. doi: 10.1007/BF00648343

Lu, H. R., Yan, G.-X., and Gallacher, D. J. (2013). A new biomarker-index of cardiac electrophysiological balance (iCEB)-plays an important role in drug-induced cardiac arrhythmias: beyond QT-prolongation and Torsades de Pointes (TdPs). J. Pharmacol. Toxicol. Methods 68, 250-259. doi: 10.1016/j.vascn.2013.01.003

Nakamura, H., Kurokawa, J., Bai, C.-X., Asada, K., Xu, J., Oren, R. V., et al. (2007). Progesterone regulates cardiac repolarization through a nongenomic pathway: an in vitro patch-clamp and computational modeling study. Circulation 116, 2913-2922. doi: 10.1161/CIRCULATIONAHA.107.702407

Odening, K. E., Choi, B. R., Liu, G. X., Hartmann, K., Ziv, O., Chaves, L., et al. (2012). Estradiol promotes sudden cardiac death in transgenic long QT type 2 rabbits while progesterone is protective. Heart Rhythm 9, 823-832. doi: 10.1016/ j.hrthm.2012.01.009

Olofsen, E., Sleigh, J. W., and Dahan, A. (2008). Permutation entropy of the electroencephalogram: a measure of anaesthetic drug effect. Br. J. Anaesth. 101, 810-821. doi: 10.1093/bja/aen290

Orosz, A., Baczkó, I., Nagy, V., Gavallér, H., Csanády, M., Forster, T., et al. (2015). Short-term beat-to-beat variability of the QT interval is increased and correlates with parameters of left ventricular hypertrophy in patients with hypertrophic cardiomyopathy. Can. J. Physiol. Pharmacol. 93, 765-772. doi: 10.1139/cjpp2014-0526

Pan, J., and Tompkins, W. J. (1985). A real-time QRS detection algorithm. IEEE Trans. Biomed. Eng. 32, 230-236. doi: 10.1109/TBME.1985.325532

Panikkath, R., Reinier, K., Uy-Evanado, A., Teodorescu, C., Hattenhauer, J., Mariani, R., et al. (2011). Prolonged Tpeak-to-tend interval on the resting ECG is associated with increased risk of sudden cardiac death. Circ. Arrhythm. Electrophysiol. 4, 441-447. doi: 10.1161/CIRCEP.110.960658

Park, B., and Lee, Y.-J. (2018). Metabolic syndrome and its components as risk factors for prolonged corrected QT interval in apparently healthy Korean men and women. J. Clin. Lipidol. 12, 1298-1304. doi: 10.1016/j.jacl.2018.07.004

Peng, C. K., Buldyrev, S. V., Havlin, S., Simons, M., Stanley, H. E., and Goldberger, A. L. (1994). Mosaic organization of DNA nucleotides. Phys. Rev. E Stat. Phys. Plasmas Fluids Relat. Interdiscip. Topics 49, 1685-1689. doi: 10.1103/PhysRevE. 49.1685

Pham, T. V., Robinson, R. B., Danilo, P., and Rosen, M. R. (2002). Effects of gonadal steroids on gender-related differences in transmural dispersion of L-type calcium current. Cardiovasc. Res. 53, 752-762. doi: 10.1016/s00086363(01)00449-7

Press, W. H., and Rybicki, G. B. (1989). Fast algorithm for spectral analysis of unevenly sampled data. Astrophys. J. 338, 277. doi: 10.1086/167197

Redfern, W. S., Carlsson, L., Davis, A. S., Lynch, W. G., MacKenzie, I., Palethorpe, S., et al. (2003). Relationships between preclinical cardiac electrophysiology, clinical QT interval prolongation and torsade de pointes for a broad range of drugs: evidence for a provisional safety margin in drug development. Cardiovasc. Res. 58, 32-45. doi: 10.1016/s0008-6363(02)00846-5
Roden, D. M. (1998). Taking the "idio" out of "idiosyncratic": predicting torsades de pointes. Pacing Clin. Electrophysiol. 21, 1029-1034. doi: 10.1111/j.1540-8159. 1998.tb00148.x

Rodriguez, I., Kilborn, M. J., Liu, X. K., Pezzullo, J. C., and Woosley, R. L. (2001). Drug-induced QT prolongation in women during the menstrual cycle. J. Am. Med. Assoc. 285, 1322-1326. doi: 10.1001/jama.285.10.1322

Rosso, O. A., Larrondo, H. A., Martin, M. T., Plastino, A., and Fuentes, M. A. (2007). Distinguishing noise from chaos. Phys. Rev. Lett. 99:154102. doi: 10. 1103/PhysRevLett.99.154102

Scargle, J. D. (1982). Studies in astronomical time series analysis. II-statistical aspects of spectral analysis of unevenly spaced data. Astrophys. J. 263:835. doi: $10.1086 / 160554$

Schwartz, P. J., and Woosley, R. L. (2016). Predicting the unpredictable: druginduced QT prolongation and torsades de pointes. J. Am. Coll. Cardiol. 67, 1639-1650. doi: 10.1016/j.jacc.2015.12.063

Shen, M. J., and Zipes, D. P. (2014). Role of the autonomic nervous system in modulating cardiac arrhythmias. Circ. Res. 114, 1004-1021. doi: 10.1161/ CIRCRESAHA.113.302549

Sims, C., Reisenweber, S., Viswanathan, P. C., Choi, B.-R., Walker, W. H., and Salama, G. (2008). Sex, age, and regional differences in L-type calcium current are important determinants of arrhythmia phenotype in rabbit hearts with drug-induced long QT type 2. Circ. Res. 102, e86-e100. doi: 10.1161/ CIRCRESAHA.108.173740

Smoczynska, A., Beekman, H. D. M., and Vos, M. A. (2019). The increment of short-term variability of repolarisation determines the severity of the imminent arrhythmic outcome. Arrhythm. Electrophysiol. Rev. 8, 166-172. doi: 10.15420/ aer.2019.16.2

Soydinc, S., Davutoglu, V., and Akcay, M. (2006). Uncomplicated metabolic syndrome is associated with prolonged electrocardiographic QTc interval and QTc dispersion. Ann. Noninvasive Electrocardiol. 11, 313-317. doi: 10.1111/j. 1542-474X.2006.00123.X

Szabó, Z., Harangi, M., Lörincz, I., Seres, I., Katona, E., Karányi, Z., et al. (2005). Effect of hyperlipidemia on QT dispersion in patients without ischemic heart disease. Can. J. Cardiol. 21, 847-850.

Thomsen, M. B., Verduyn, S. C., Stengl, M., Beekman, J. D. M., de Pater, G., van Opstal, J., et al. (2004). Increased short-term variability of repolarization predicts d-sotalol-induced torsades de pointes in dogs. Circulation 110, $2453-$ 2459. doi: 10.1161/01.CIR.0000145162.64183.C8

Tian, J., Ai, Y., Fei, C., Zhao, M., Zhang, F., and Wang, Z. (2018). Fault diagnosis of intershaft bearings using fusion information exergy distance method. Shock Vib. 2018:7546128. doi: 10.1155/2018/7546128

Tisdale, J. E., Jaynes, H. A., Overholser, B. R., Sowinski, K. M., and Kovacs, R. J. (2019). Progesterone pretreatment reduces the incidence of druginduced torsades de pointes in atrioventricular node-ablated isolated perfused rabbit hearts. J. Cardiovasc. Electrophysiol. 30, 941-949. doi: 10.1111/jce. 13942

Tisdale, J. E., Overholser, B. R., Wroblewski, H. A., and Sowinski, K. M. (2011). The influence of progesterone alone and in combination with estradiol on ventricular action potential duration and triangulation in response to potassium channel inhibition. J. Cardiovasc. Electrophysiol. 22, 325-331. doi: 10.1111/j. 1540-8167.2010.01869.x

Unakafova, V. A. (2015). Investigating Measures of Complexity for Dynamical Systems and for Time Series. Ph. D. thesis, Lubeck: University of Lubeck.

Valentin, J. P. (2010). Reducing QT liability and proarrhythmic risk in drug discovery and development. Br. J. Pharmacol. 159, 5-11. doi: 10.1111/j.14765381.2009.00547.x

Vedre, A., Gurm, H. S., Froehlich, J. B., Kline-Rogers, E., Montalescot, G., Gore, J. M., et al. (2009). Impact of prior statin therapy on arrhythmic events in patients with acute coronary syndromes (from the Global Registry of Acute Coronary Events [GRACE]). Am. J. Cardiol. 104, 1613-1617. doi: 10.1016/j. amjcard.2009.07.045

Verduyn, S. C., Vos, M. A., Van Der Zande, J., Kulcsàr, A., and Wellens, H. J. J. (1997). Further observations to elucidate the role of interventricular dispersion of repolarization and early afterdepolarizations in the genesis of acquired tarsade de pointes arrhythmias: a comparison between almokalant and d-sotalol using the dog as its own control. J. Am. Coll. Cardiol. 30, 1575-1584. doi: 10.1016/S0735-1097(97)00333-1 
Vincze, D., Farkas, A. S., Rudas, L., Makra, P., Csík, N., Leprán, I., et al. (2008). Relevance of anaesthesia for dofetilide-induced torsades de pointes in $\alpha 1$ adrenoceptor-stimulated rabbits. Br. J. Pharmacol. 153, 75-89. doi: 10.1038/sj. bjp.0707536

Yang, X., Chen, G., Papp, R., Defranco, D. B., Zeng, F., and Salama, G. (2012). Oestrogen upregulates L-type Ca $2+$ channels via oestrogen-receptor- $\alpha$ by a regional genomic mechanism in female rabbit hearts. J. Physiol. 590, 493-508. doi: 10.1113/jphysiol.2011.219501

Yang, X., Mao, X., Xu, G., Xing, S., Chattopadhyay, A., Jin, S., et al. (2018). Estradiol up-regulates L-type Ca2+ channels via membrane-bound estrogen receptor/phosphoinositide-3-kinase/Akt/cAMP response elementbinding protein signaling pathway. Heart Rhythm 15, 741-749. doi: 10.1016/ j.hrthm.2018.01.019

Yu, Q., Li, Y., Waqar, A. B., Wang, Y., Huang, B., Chen, Y., et al. (2012). Temporal and quantitative analysis of atherosclerotic lesions in diet-induced hypercholesterolemic rabbits. J. Biomed. Biotechnol. 2012:506159. doi: 10.1155/ 2012/506159
Zhang, B., Zhang, L., Xu, J., and Liu, J. (2013). Information exergy-based method for structural damage diagnosis. J. Vibroengineering 15, 1606-1618.

Zhang, B., Zhang, L., Xu, J., and Wang, P. (2014). Performance degradation assessment of rolling element bearings based on an index combining SVD and information exergy. Entropy 16, 5400-5415. doi: 10.3390/e16105400

Conflict of Interest: The authors declare that the research was conducted in the absence of any commercial or financial relationships that could be construed as a potential conflict of interest.

Copyright (C) 2021 Nalos, Jarkovská, Švíglerová, Süß, Záleský, Rajdl, Krejčová, Kuncová, Rosenberg and Štengl. This is an open-access article distributed under the terms of the Creative Commons Attribution License (CC BY). The use, distribution or reproduction in other forums is permitted, provided the original author(s) and the copyright owner(s) are credited and that the original publication in this journal is cited, in accordance with accepted academic practice. No use, distribution or reproduction is permitted which does not comply with these terms. 